\title{
Addendum
}

\section{Neutron depolarization theory in the larmor and the scattering approach}

\author{
R. Rosman, H. Frederikze, and M.Th. Rekveldt \\ Interfacultair Reactor Instituut, Delft University of Technology, 2629 JB Delft, The Netherlands
}

Received May 25, 1990

Z. Phys. B - Condensed Matter 79, 61 (1990)

In the paper "Neutron depolarization theory in the Larmor and the scattering approach' [1], it has been shown that the extended Larmor and the scattering approach are basically equivalent but differ formally. However, both approaches are fully equivalent, which is shown in this comment. Furthermore, the equivalence of the Larmor and the extended Larmor approach in the case of infinitely elongated ellipsoids is shown in a more formal way than was done in [1]. For completeness, an inaccuracy and a printing error in two expressions of the original paper are corrected.

In [1], it has been shown that the expressions for the depolarization matrix $\hat{D}$ in the scattering and the Larmor approach are equivalent, except for a term related to the vector

$\int_{\Omega} \mathbf{q}(\boldsymbol{\kappa}) \mathrm{d} \Omega$,

with

$\mathbf{q}(\boldsymbol{\kappa})=\int_{\Delta V} \mathrm{~d}^{3} \mathbf{r} \int_{\Delta V} \mathrm{~d}^{3} \mathbf{r}^{\prime} \mathrm{e}^{i \boldsymbol{\kappa} \cdot\left(\mathbf{r}-\mathbf{r}^{\prime}\right)} \mathbf{M}^{*}\left(\tilde{\boldsymbol{\kappa}}, \mathbf{r}^{\prime}\right) x \mathbf{M}^{*}(\tilde{\boldsymbol{\kappa}}, \mathbf{r})$

$\mathbf{M}^{*}(\tilde{\boldsymbol{\kappa}}, \mathbf{r})=\tilde{\boldsymbol{\kappa}} x(\mathbf{M}(\mathbf{r}) x \tilde{\boldsymbol{\kappa}})$.

Here, $\Delta V$ is a part of the medium considered, $\mathbf{M}(\mathbf{r})$ the local magnetization, $\boldsymbol{\kappa}$ the scattering vector, $\tilde{\boldsymbol{\kappa}}=\boldsymbol{\kappa} /|\boldsymbol{\kappa}|$, $\mathrm{d} \Omega=\frac{\lambda^{2}}{4 \pi^{2}} \mathrm{~d} \boldsymbol{\kappa}$ and $\lambda$ the neutron wavelength. Obviously, $\mathbf{q}(\boldsymbol{\kappa})$ is an anti-symmetric function of $\boldsymbol{\kappa}(\mathbf{q}(\boldsymbol{\kappa})=-\mathbf{q}(-\boldsymbol{\kappa}))$. Therefore, the contribution of the term in a neutron transmission experiment, i.e. after integration over $\Omega$ under the approximation that $\kappa_{z}=0$, vanishes for any $\mathbf{M}(\mathbf{r})$ distribution. This implies the full equivalence of the extended Larmor and the scattering approach. As the term discussed is not present in the formulas derived by Maleev and Ruban in the scattering approach [2, 3], they probably used the same argument.
The equivalence of the Larmor and the extended Larmor approach in the case of infinitely elongated ellipsoids can be proved in a more formal and also simpler way than has been done in [1] (31). The equivalence directly follows from a comparison of the general expressions for $\alpha_{i j}$ of ellipsoids with axial dimension $2 b$, radial dimension $2 a$ and a spontaneous magnetization $\mathbf{M}$ along the $b$-axis in both approaches. In the extended Larmor approach this expression is given by (see (29) of [1])

$\alpha_{i j}=\delta_{i j}\left(\mu_{0} M\right)^{2} \frac{3 b}{8 \pi} \int_{\tilde{S}} \mathrm{~d} \tilde{\mathbf{s}}\left\langle\frac{\left(n_{i}^{*}(\tilde{\mathbf{s}})\right)^{2}}{c(\mathbf{n}, \tilde{\mathbf{s}})}\right\rangle$

with

$$
\begin{aligned}
& \mathbf{n}^{*}(\tilde{\mathbf{s}})=\tilde{\mathbf{s}} x(\mathbf{n} \times \tilde{\mathbf{s}}) \\
& c(\mathbf{n}, \tilde{\mathbf{s}})=(\mathbf{n} \cdot \tilde{\mathbf{s}})^{2}\left(\left(\frac{b}{a}\right)^{2}-1\right)+1 .
\end{aligned}
$$

Here, $\mathbf{n}$ is the unit vector along $\mathbf{M},\langle>$ an average over all ellipsoids and $\widetilde{S}$ the $s_{x} s_{y}$-plane. The corresponding expression in the Larmor approach is given by (2) with $\left(n_{i}(\mathbf{\mathbf { s }})\right)^{2}$ replaced by $n_{i}^{2}$. In the limit of $b / a$ approaching infinity, only those $\tilde{\mathbf{s}}$-vectors for which $(\mathbf{n} \cdot \tilde{\mathbf{s}})=0$ contribute to $\alpha_{i j}$. For these vectors $\mathbf{n}^{*}(\tilde{\mathbf{s}})$ equals $\mathbf{n}$, as a consequence of which the expressions for $\alpha_{i j}$ in the Larmor and the extended Larmor approach are equivalent. Note that (31) of [1] contains an inaccuracy. Its right-handside should be multiplied by $9 / 8$. Furthermore, it contains a printing error. The correct version is

$$
\alpha_{x x}=\alpha_{y y}=\left(\mu_{0} M\right)^{2} \frac{3 a}{8}\left[\frac{\frac{1}{2}\left(\theta_{2}-\theta_{1}\right)-\frac{1}{4}\left(\sin 2 \theta_{2}-\sin 2 \theta_{1}\right)}{\cos \theta_{1}-\cos \theta_{2}}\right]
$$

$$
a_{z z}=\left(\mu_{0} M\right)^{2} \frac{3 a}{8}\left[\frac{\left(\theta_{2}-\theta_{1}\right)+\frac{1}{2}\left(\sin 2 \theta_{2}-\sin 2 \theta_{1}\right)}{\cos \theta_{1}-\cos \theta_{2}}\right] .
$$


Also (28) of [1], expressing the equation for $\alpha_{i i}$ of an isotropic distribution of ellipsoids in the Larmor approach, needs some correction: the integrand on its right-hand-side should be multiplied with $2 \sin (\theta)$. After some rewriting, the correct version of (28) reads

$$
\begin{aligned}
\alpha_{i i}= & \left(\mu_{0} M\right)^{2} \int_{0}^{\pi / 2}\left[\left(\delta_{i x}+\delta_{i y}\right) \sin ^{2}(\theta) / 2+\delta_{i z} \cos ^{2}(\theta)\right] \\
& \cdot f(\theta, a, b) \mathrm{d} \theta
\end{aligned}
$$

with

$$
f(\theta, a, b)=\frac{3}{4} b \tan (\theta) /\left[1+\left(\frac{b}{a}\right)^{2} \tan ^{2}(\theta)\right]^{1 / 2} .
$$

\section{References}

1. Rosman, R., Rekveldt, M.Th.: Z. Phys. B - Condensed Matter 79, 61 (1990)

2. Maleev, S.V., Ruban, V.A.: JETP 35 (1), 222 (1972)

3. Maleev, S.V., Ruban, V.A.: Sov. Phys. Solid State 18 (8), 1331 (1976) 